\title{
ASOCIACIONISMO EN RED PARA LA PLANIFICACIÓN DEL DESARROLLO TURÍSTICO SOSTENIBLE. EL CASO DEL RÍO GUADALQUIVIR Y SUS PRINCIPALES AFLUENTES ${ }^{1}$.
}

\section{NETWORK BUILDING AS A TOOL FOR SUSTAINABLE TOURISM DEVELOPMENT PLANNING. THE GUADALQUIVIR RIVERSIDE AND ITS MAIN TRIBUTARIES CASE STUDY.}

\author{
Gustavo A. Contreras Cabrera \\ Universidad de Sevilla \\ gcontreras@us.es
}

Recibido: febrero, 2012.

Versión final aceptada: noviembre, 2012.

PALABRAS-CLAVE: asociacionismo en red, desarrollo local, espacios naturales protegidos, turismo sostenible.

KEY WORDS: network associations, local development, natural protected areas, sustainable tourism.

RESUMEN:

A partir del año 2007 se ha producido en Andalucía un complejo proceso de cooperación institucional en red para promover el desarrollo local sostenible en el ámbito territorial del Guadalquivir. Esta situación se fundamenta en la voluntad política de impulsar el desarrollo socioeconómico de sus municipios ribereños, mediante la puesta en valor del patrimonio existente. Sus principales hitos han sido la creación de la Asociación Red de Ciudades Río Guadalquivir y el posterior Consorcio Guadalquivir Turístico, que incorpora el eje secundario del río Genil.

\footnotetext{
1 El presente artículo procede de la comunicación científica presentada en las Jornadas de Estudios Regionales: Redes y sus territorios: Incidencia en el desarrollo regional. AGE. Sevilla, Junio de 2012.
}

ISSN: 0212-8594 ISSN-e: 2340-2776 № DOI: http://dx.doi.org/10.12795/rea.2011.i28.03

REA 28 (2011):34-54

http://www.publius.us.es/estudios_andaluces 
Este artículo recoge esos antecedentes, avanzando en la posibilidad de considerar la especial condición del río Guadalquivir y otros afluentes como corredores ecológicos entre espacios naturales de alto valor para el inicio de una estrategia de desarrollo turístico sostenible en red.

\section{ABSTRACT:}

Since the year 2007, there has been a complex process of institutional cooperation in Andalusia, that brought about a local institutional network, to promote local sustainable development in the Guadalquivir riverside. This process succeeded thanks to the political will of the riparian municipalities, to promote the socioeconomic development through preserving and promoting their cultural and natural heritage. Their major feat has been the creation of the Riparian Cities of Guadalquivir Network Association, eventually transformed to the Guadalquivir Tourist Consortium, which includes the Genil river also.

This research paper reflects on this background, and realizes the strategic role of the Guadalquivir River and some of its tributaries as ecological corridors, that connect protected areas of high value. Accordingly, the introduction of these issues in a strategy for a sustainable tourist development network, could improve its efficiency. 


\section{INTRODUCCIÓN.}

Desde el año 2007 se ha experimentado en Andalucía un creciente proceso de cooperación intermunicipal en red para promover el desarrollo local sostenible en el ámbito territorial del río Guadalquivir (Fernández, F.M., 2012). Este proceso ha sido consecuente con la voluntad política de dar impulso al desarrollo socioeconómico de sus municipios ribereños, a través de la puesta en valor del rico patrimonio natural, cultural e histórico existente (Alburquerque, F. y Delgadillo, J., 2009).

Los hitos más destacados en este caso de asociacionismo en red han sido la constitución de la Asociación Red de Ciudades Río Guadalquivir (marzo de 2007) y el posterior Consorcio Guadalquivir Turístico (mayo de 2011), cuyas características y objetivos serán considerados más adelante.

En este contexto, el turismo sostenible se perfila como una estrategia de alto impacto en el empleo y en el desarrollo endógeno de unos municipios que, desde hace años, han protagonizado experiencias mayoritariamente a escala local y con diferentes niveles de éxito (Cantarero, J., 2001). La unificación de esfuerzos y recursos en torno a un producto turístico con atractivo resultará esencial para alcanzar mayores cotas de mercado en un contexto económico innovador, en permanente transformación y con fuerte competitividad (Palenzuela, P., 2000). Por consiguiente, el objetivo fundamental de este estudio consiste en verificar la viabilidad de las especiales condiciones del río Guadalquivir y sus afluentes como aglutinadoras de una estrategia regional de desarrollo turístico sostenible.

\section{ANTECEDENTES.}

Existen dos importantes antecedentes en el aprovechamiento del Guadalquivir como recurso para el impulso socioeconómico sostenible de los municipios ribereños:

- Iniciativas aisladas, impulsadas especialmente desde las grandes ciudades enclavadas junto a este eje fluvial, y/o desde organismos o entidades instrumentales vinculadas al turismo.

- Iniciativa asociativa común basada en una red de municipios que aúnan esfuerzos para aumentar su capacidad de tractivo y generar efectos multiplicadores en sus acciones o inversiones.

Esta segunda opción se corresponde con la creación de la Asociación Red de Ciudades Río Guadalquivir, en marzo de 2007, integrada por 35 municipios ribereños de este río (13 de la provincia de Sevilla, 11 de Jaén, 9 de Córdoba, y 2 de Cádiz): 
Gustavo A. Contreras Cabrera

Asociacionismo en red para la planificación del desarrollo turístico sostenible. El caso del río Guadalquivir y sus principales afluentes

Figura 1. Entidades asociadas a la Red.

\begin{tabular}{|c|c|}
\hline Provincias & Municipios \\
\hline Cádiz & $\begin{array}{c}\text { Sanlúcar de Barrameda } \\
\text { Trebujena }\end{array}$ \\
\hline Córdoba & $\begin{array}{c}\text { Adamuz } \\
\text { Córdoba } \\
\text { El Carpio } \\
\text { Montoro } \\
\text { Palma del Río } \\
\text { Pedro Abad } \\
\text { Posadas } \\
\text { Villa del Río } \\
\text { Villafranca de Córdoba }\end{array}$ \\
\hline Jaén & $\begin{array}{c}\text { Baeza } \\
\text { Cazorla } \\
\text { Iznatoraf } \\
\text { Lopera } \\
\text { Mancha Real } \\
\text { Marmolejo } \\
\text { Quesada } \\
\text { Santo Tomé } \\
\text { Úbeda } \\
\text { Villacarrillo } \\
\text { Villanueva de la Reina }\end{array}$ \\
\hline Sevilla & $\begin{array}{c}\text { Alcolea del Río } \\
\text { Cantillana } \\
\text { Coria del Río } \\
\text { Dos Hermanas } \\
\text { Gelves } \\
\text { La Puebla del Río } \\
\text { La Rinconada } \\
\text { Lora del Río } \\
\text { Peñaflor } \\
\text { Sevilla } \\
\text { Tocina } \\
\text { Villanueva del Río y Minas } \\
\text { Villaverde del Río }\end{array}$ \\
\hline
\end{tabular}

Fuente: Elaboración propia, a partir de los Estatutos de la Red de Ciudades del Río Guadalquivir. 
La Red de Ciudades fija entre sus fines la puesta en marcha conjunta de estrategias y acciones promocionales dirigidas a operadores turísticos y otros profesionales para la difusión de la imagen del río Guadalquivir como soporte de la actividad turística a desarrollar por los miembros asociados, realizando una gestión sostenible de los activos del medio físico por medio de la conservación, restauración, recreación y aprovechamiento social de los recursos que se corresponda con los intereses de los municipios de la Red. También, se optó por la planificación y desarrollo de una política de producción y promoción turística y de difusión de imagen, compatible con los intereses de estos municipios, con la idea de optimizar la demanda turística.

Estas previsiones se apuntalan con la propuesta común de iniciar gestiones ante la UNESCO para la declaración del Río Guadalquivir como Patrimonio de la Humanidad.

La constitución de la Red de Ciudades y la aprobación de sus estatutos de organización y funcionamiento coincidieron con la celebración de unas primeras Jornadas de trabajo y debate centradas en los objetivos y prioridades de la entidad, y en la redacción de unas conclusiones y un calendario de actividades prioritarias, como la fijación de un Plan Anual de Actuaciones para 2008.

Figura 2. Acto protocolario de aprobación del Acta fundacional (Sevilla, 14 de marzo de 2007).

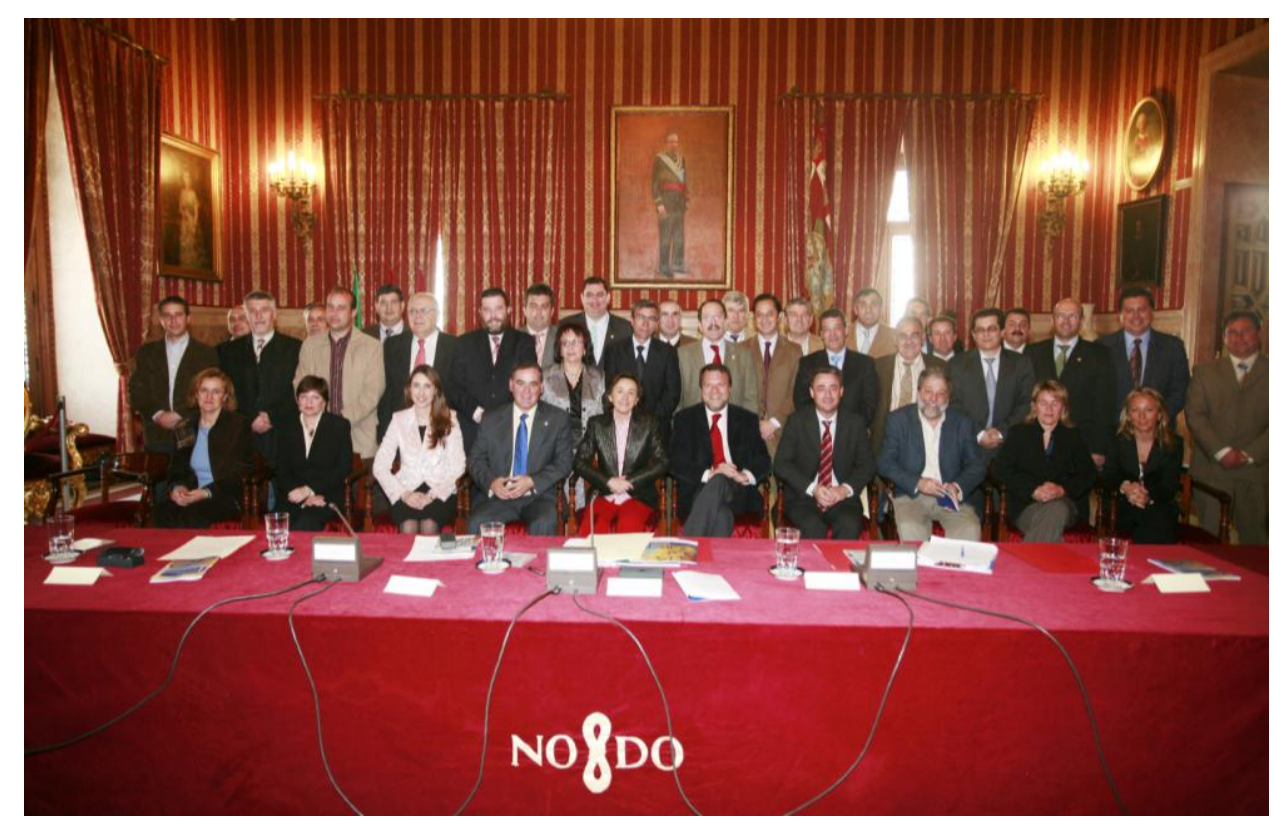

Fuente: Red de Ciudades del río Guadalquivir (2007).

Un año después, se produce un hecho relevante para esta red, derivado del discurso de investidura del nuevo presidente de la Comunidad Autónoma de Andalucía. En dicho evento se fijan 108 compromisos, entre los que se incluye la "Creación de la ruta turística del interior" (compromiso 27) ${ }^{2}$. Este compromiso prevé la implementación de

\footnotetext{
${ }^{2}$ Ver en http://www.juntadeandalucia.es/compromisos20082012/principal_medida.php?id_medida=21
} 
esa ruta para la puesta en valor de las ciudades, espacios y productos que inciden en torno al eje central de este río. Todo ello, a través del proyecto "Guadalquivir Turístico", que supondría un tratamiento más integral del impulso turístico en este ámbito territorial, optimizando otras experiencias individuales iniciadas, como los planes turísticos de Sevilla, de las Riberas del Guadaira, del Río Guadalquivir o las iniciativas de Turismo sostenible del Valle del Guadalquivir y de la Vega del Guadalquivir.

Según recogía la prensa en 2009, este proyecto sobrepasaba la dimensión empresarial o de generación de empleo, al tratar de dibujar un nuevo modelo socioeconómico en los municipios beneficiarios. Un efecto resultante de la aplicación del proyecto sería la reducción en un $40 \%$ de la emigración interior de los pueblos de la cuenca hacia las grandes ciudades o la costa andaluza (la Estadística de Variaciones Residenciales del Instituto de Estadística de Andalucía reflejaba en ese año un cambio de residencia en más de 165.000 ciudadanos entre las provincias andaluzas. De éstas, casi el $80 \%$ procedía de municipios del valle del Guadalquivir).

Figura 3. Presentación del Proyecto Guadalquivir Turístico en la Feria Internacional de Turismo (FITUR) 2010.

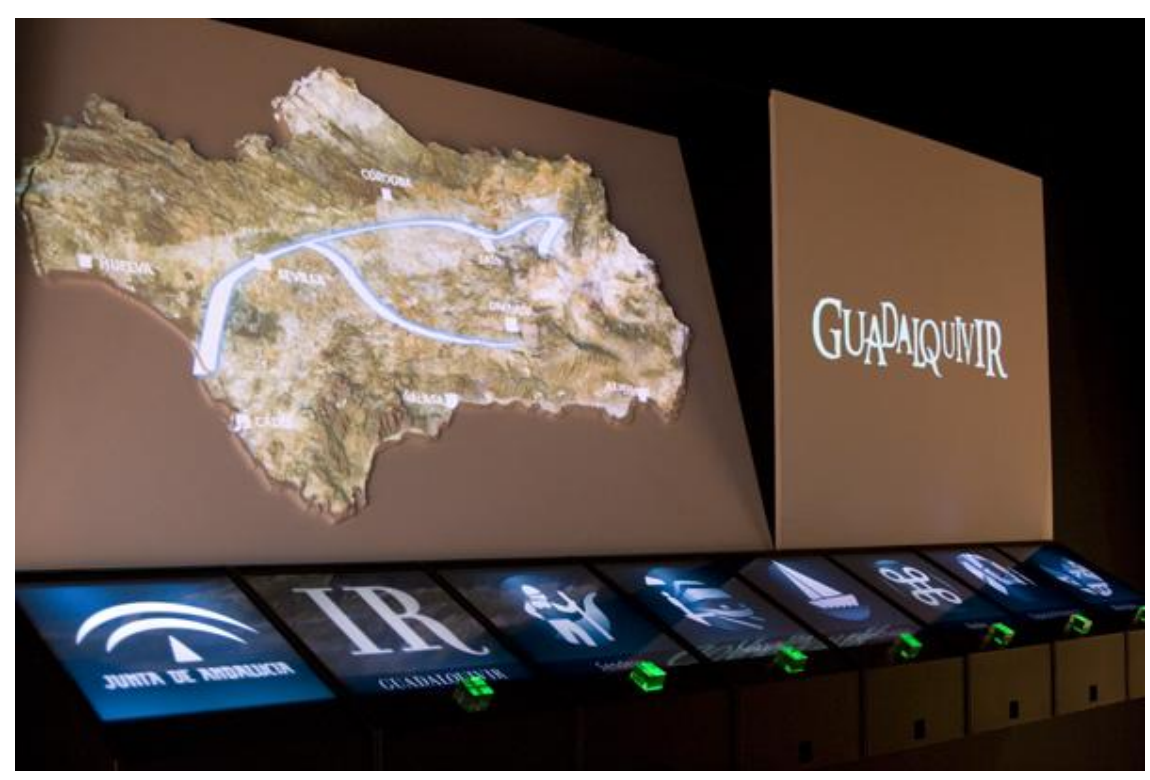

Fuente: http://www.andaluciaturismodigital.com/galeria-fitur2010.asp

El reto autonómico de crear esta ruta turística de interior y su implementación a través de un proyecto definido supone para la Red de Ciudades un importante estímulo, aunque también es preciso aplicar una fórmula válida que dote de operatividad la interacción de dos realidades administrativas diferentes, como son la Autonómica y la Local, además de los agentes sociales y económicos miembros del Consejo Económico y Social de Andalucía. Se opta por la creación de un Consorcio impulsado mediante acuerdo del Consejo de Gobierno de la Junta de Andalucía en mayo de 2011, cuya composición definitiva de miembros asociados es la siguiente: 
a) Junta de Andalucía (Consejerías competentes en materia de Presidencia, Economía y Hacienda, Obras Públicas y Transportes, Vivienda y Ordenación del Territorio, Turismo, Comercio y Deporte, Cultura y Medio Ambiente).

b) Diputaciones provinciales de Cádiz, Córdoba, Granada, Huelva, Jaén y Sevilla.

c) Red de Ciudades Río Guadalquivir.

d) Confederación de Empresarios de Andalucía.

e) Sindicatos Comisiones Obreras-Andalucía (CCOO-A) y Unión General de Trabajadores-Andalucía (UGT-A).

Así mismo, se estimó conveniente la ampliación del ámbito geográfico inicialmente cubierto por la Red de Ciudades, incorporando el eje del río Genil en lo que se vino a denominar como la "Y andaluza".

Con la denominación "Guadalquivir Turístico", este consorcio expresa en el artículo 3 de sus estatutos las siguientes intenciones:

a) Fomentar el desarrollo de actuaciones integrales vinculadas al río basadas en la diferenciación a partir del reforzamiento de la identidad del espacio turístico utilizando como valores de distinción el patrimonio natural y paisajístico, el patrimonio y legado cultural de cada producto, y los criterios de ordenación territorial fijados por la Administración andaluza.

b) Articular mecanismos de cooperación económica, técnica y administrativa entre las diferentes Administraciones y entidades públicas y privadas con competencias o actuaciones en el ámbito del río Guadalquivir a fin de ejercer de forma conjunta y coordinada las competencias que les corresponden en materia de gestión, desarrollo y consolidación de la competitividad del río Guadalquivir como destino turístico.

c) Impulsar y fomentar las iniciativas públicas y privadas dirigidas a la provisión de equipamientos y servicios en continua adaptación a las demandas específicas del turismo vinculado con la cuenca del Guadalquivir y su área de influencia.

d) Ejecutar las obras relativas a la construcción de equipamientos, dotaciones e instalaciones que se consideren convenientes, así como las eventuales encomiendas de gestión que se practiquen a favor del Consorcio por los entes consorciados o por aquellas otras instituciones públicas que así lo acuerden.

e) Fomentar el diseño de la oferta de actividades con una doble orientación poblacional. Atendiendo a las necesidades de ocio del público de las poblaciones del entorno, así como a la demanda turística específica y segmentada de los mercados objeto de este Plan. 
f) Impulsar actuaciones para concienciar y sensibilizar a toda la población afectada por esta iniciativa y sea entendida como un proyecto común y una verdadera oportunidad generadora de empleo y riqueza para su territorio.

La integración de la Red de Ciudades Río Guadalquivir en Guadalquivir Turístico supone una pérdida importante de identidad municipalista del proyecto asociativo inicial, como lo demuestra su participación en el capital fundacional que se establece (2 millones de euros): Junta de Andalucía, el 53\%; Diputaciones provinciales asociadas, $36 \%$; Red de Ciudades, $5 \%$; la CEA, $3 \%$; y los sindicatos, $3 \%$. No obstante, este cambio representa la disponibilidad de un mayor volumen de recursos técnicos y económicos que serán garantizados, en gran parte, a través del presupuesto anual que se apruebe en su Consejo Rector, además de elaborar un plan de trabajo basado en la coordinación y la cooperación de los agentes consorciados en línea con los fines de la Red.

Esta nueva figura asociativa en red establece tres herramientas básicas para llevar a cabo sus propósitos constitutivos: Un Programa general de Actuaciones, un Plan Anual de Actuación y un Presupuesto para cada ejercicio económico.

El Programa de Actuaciones representa la directriz estratégica diseñada para la consecución de los fines mencionados, teniendo en cuenta para la asignación de recursos variables tan importantes como la población, la oferta turística y la extensión del espacio de interés turístico, así como el acuerdo de transferir competencias y servicios por parte de las Administraciones competentes en cada caso (a través de encomienda expresa al Consorcio).

La puesta en marcha del Programa de Actuación de Guadalquivir Turístico representa la movilización de una importante inversión público-privada dirigida a la construcción y/o adaptación de equipamientos e infraestructuras para el ocio y la creación de nuevos productos turísticos, culturales, y deportivos en el ámbito del Guadalquivir y el Genil. Así mismo, se pretende impulsar la economía y generar empleo a través de acciones de mejora de vías de comunicación y medios de transporte, el fomento de la navegabilidad de estos ríos, la recuperación del patrimonio hidrológico, la capacitación y apoyo a emprendedores o la puesta en valor con criterios de sostenibilidad de espacios naturales y urbanos.

\section{OPORTUNIDADES PARA LA ACCIÓN SOSTENIBLE EN RED: INVESTIGAR EL DESARROLLO RURAL SOSTENIBLE VINCULADO EL RÍO GUADALQUIVIR Y SUS PRINCIPALES AFLUENTES.}

Junto a las ideas y antecedentes expuestos en los apartados anteriores, es posible avanzar en la posibilidad de considerar la especial condición del río Guadalquivir y otros afluentes como corredores ecológicos entre espacios naturales protegidos (EPN) de alto valor añadido para la puesta en marcha de una estrategia de desarrollo local sostenible en red que beneficie a los municipios que forman parte de este contexto geográfico (Contreras, G. A. y Jiménez, E., 2010). 
La incorporación al ámbito geográfico de partida de otros afluentes, además del río Genil, representa una innovación interesante surgida a finales de 2010 entre un grupo de investigadores del Instituto de Desarrollo Regional (IDR), fundación vinculada a la Universidad de Sevilla.

En este sentido, se consideró por parte del IDR, la oportunidad de ofrecer a los poderes públicos competentes un modelo de planificación relacionado con la sostenibilidad del desarrollo turístico citado, aprovechando la exitosa experiencia acumulada de su Línea de Investigación "Economía, territorio, medio ambiente y desarrollo sostenible" para elaborar una propuesta de planificación participativa.

Aunque antes de crearse la figura del Consorcio Guadalquivir Turístico y su Programa de Actuación se había iniciado una campaña institucional de presentación y respaldo a esta idea por parte de la Administración Autonómica de Andalucía (octubre de 2009), adelantando líneas de actuación y acciones concretas, como la ruta de senderismo, a modo de vía verde, paralela al río (de 700 kilómetros de longitud), la remodelación de 33 estaciones de tren existentes en este ámbito para la puesta en marcha del proyecto denominado "Guadalrail", o la creación de 42 estaciones fluviales en tramos de río y embalses como zonas de uso recreativo, atribuyendo a 3 de éstas estaciones el carácter de "Grandes Nodos" del río: Nodo del Guadalquivir Alto o ventana de la Sierra, Nodo o ventana de a Vega y Nodo o ventana de la Marisma, desde el IDR se apreció la posibilidad de aplicar un modelo de planificación basado en dos pilares interesantes:

- Apertura de un proceso participativo de toma de opiniones, ideas y propuestas concretas entre la población de los municipios incluidos en el ámbito general seleccionado. Especialmente, se consideró clave la toma de opinión de los agentes más representativos de los territorios beneficiarios, como los gestores locales, las entidades asociativas sin ánimo de lucro presentes, el empresariado y los agentes sociales.

- Incorporación de la figura de los espacios naturales protegidos más cercanos al río Guadalquivir, entendiendo a éste como un corredor ecológico de primer orden capaz de conectar a espacios de especial valor natural y con atractivo suficiente para provocar un efecto multiplicador en el desarrollo turístico perseguido para este contexto geográfico.

Estas cuestiones tuvieron cabida en la apertura de un proceso de investigación dirigido hacia un "Plan de Acción para el Desarrollo Rural Sostenible vinculado el río Guadalquivir y sus principales afluentes". Se pretendía con ello, mostrar una nueva vía de planificación basada en una confluencia de aportes metodológicos puestos en práctica por parte de esta Fundación, como los enfoques del Marco Lógico para la planificación de proyectos orientada mediante objetivos (Fundación centro Español de Estudios de América Latina, 1997), la dinamización de agentes o actores locales, a través de talleres y técnicas participativas, o el adelanto de niveles suficientes de 
racionalidad y coherencia en el proceso de planificación, como garantía de una evaluación conceptual o de diseño positiva.

\subsection{OBJETIVOS DEL PLAN.}

El diseño de este Plan de Acción tendría como Objetivo General anticipar estrategias de desarrollo integral sostenible a los municipios andaluces vinculadas a las nuevas condiciones medioambientales del río Guadalquivir y sus principales afluentes, mediante la puesta en valor de sus recursos patrimoniales:

- Patrimonio natural (paisaje, espacios protegidos, ecosistemas bien conservados, fauna y, flora) asociado a la Red Natura 2000 y la Red de Espacios Naturales Protegidos de Andalucía (RENPA).

- Patrimonio histórico y monumental.

- Patrimonio cultural-etnológico (fiestas, costumbres y tradiciones).

Así mismo, se parte de un nuevo contexto de desarrollo, en el cual el río Guadalquivir y sus principales afluentes adquieren un significado distinto para las políticas medioambientales de Andalucía en el marco de la Unión Europea: Como espacios sometidos a nuevo estatus de protección (Red Natura 2000) y como corredores ecológicos entre espacios de la RENPA ya consolidados (García y Montes (2003). Dicha situación requiere una concreción de estrategias de desarrollo sostenible integradas que reorienten las condiciones existentes de desarrollo socioeconómico en los municipios integrados (Galiana, L. y Vinuesa, J., 2010).

Esta reflexión resulta convincente para la definición de cuatro Objetivos Específicos o de desarrollo del Objetivo General expuesto:

- Escenario de Partida: Definición del escenario de partida (prospección de las dimensiones social, económica y medioambiental del territorio).

- Diagnóstico Participativo: Participación socio-institucional en la detección de potencialidades más destacadas del río Guadalquivir y sus afluentes seleccionados, para el desarrollo rural sostenible de este ámbito geográfico.

- Plan de Acción: Diseño de un Plan de Acción Integrada como estrategia para el desarrollo rural sostenible en los municipios del área de influencia socioeconómica3 del Guadalquivir y sus principales afluentes.

\footnotetext{
${ }^{3}$ La utilización del término "área de influencia socioeconómica" obedece a un intento de equipararlo metodológicamente con su significado y aplicación en los espacios naturales protegidos: Con el fin de contribuir al mantenimiento de los EPN y compensar socioeconómicamente a las poblaciones locales asentadas, se declaran como áreas de influencia socioeconómica al conjunto de términos municipales donde se ubican dichos espacios o sus correspondientes zonas periféricas de protección, si procede.
} 
- Integración de los EPN: Integración de la Red NATURA 2000 y RENPA como referentes fundamentales del desarrollo sostenible de estos espacios.

La integración de los espacios naturales protegidos como referentes del desarrollo sostenible en red viene avalada por su fuerte atractivo en la promoción turística de interior y su presencia en diversos tramos de los ríos Guadalquivir y Genil, así como en sus proximidades.

\subsection{CARACTERÍSTICAS DEL PLAN DE ACCIÓN.}

El avance de investigación en torno al diseño de este Plan de Acción contempla tres características de interés, cuyos principales valores son la determinación de su identidad:

- Impulsor de una mejor percepción socio-institucional del Guadalquivir y sus afluentes principales como componentes claves para el desarrollo sostenible del territorio mediante una estrategia participativa e integral.

- Establece prioridades en el tiempo y en el espacio.

- Define los medios necesarios, el reparto de funciones y los métodos de seguimiento y evaluación de actuaciones.

\subsection{DELIMITACIÓN DEL ÁMBITO DE ESTUDIO.}

Por cuestiones operativas se propuso la zonificación del ámbito de estudio en función de los Grupos de Desarrollo Rural ubicados en las áreas de influencia socioeconómica del río Guadalquivir y varios afluentes de interés.

Los afluentes elegidos para este trabajo son el Genil, la Madre de las Marismas del Rocío, el Guadiamar, la Ribera de Huelva, el Bembézar, el Guadiato, el Jándula y el Guadiana Menor, resultando ser una primera aproximación al modelo de corredores ecológicos con espacios naturales de importancia muy definida. No obstante, sería lógica y razonable una posterior ampliación a otros afluentes de similar relevancia, como la Ribera de Huésna, el Corbones, el Retortillo o el Guadalimar.

Además, fue planteada una labor concordante con las condiciones y criterios ambientales propios de los Lugares de Importancia Comunitaria (LIC) de la Red NATURA 2000 y de la RENPA existentes, según su estatus de espacios protegidos o de pasillos ecológicos. 
Figura 4: El río Guadalquivir y sus principales afluentes seleccionados.

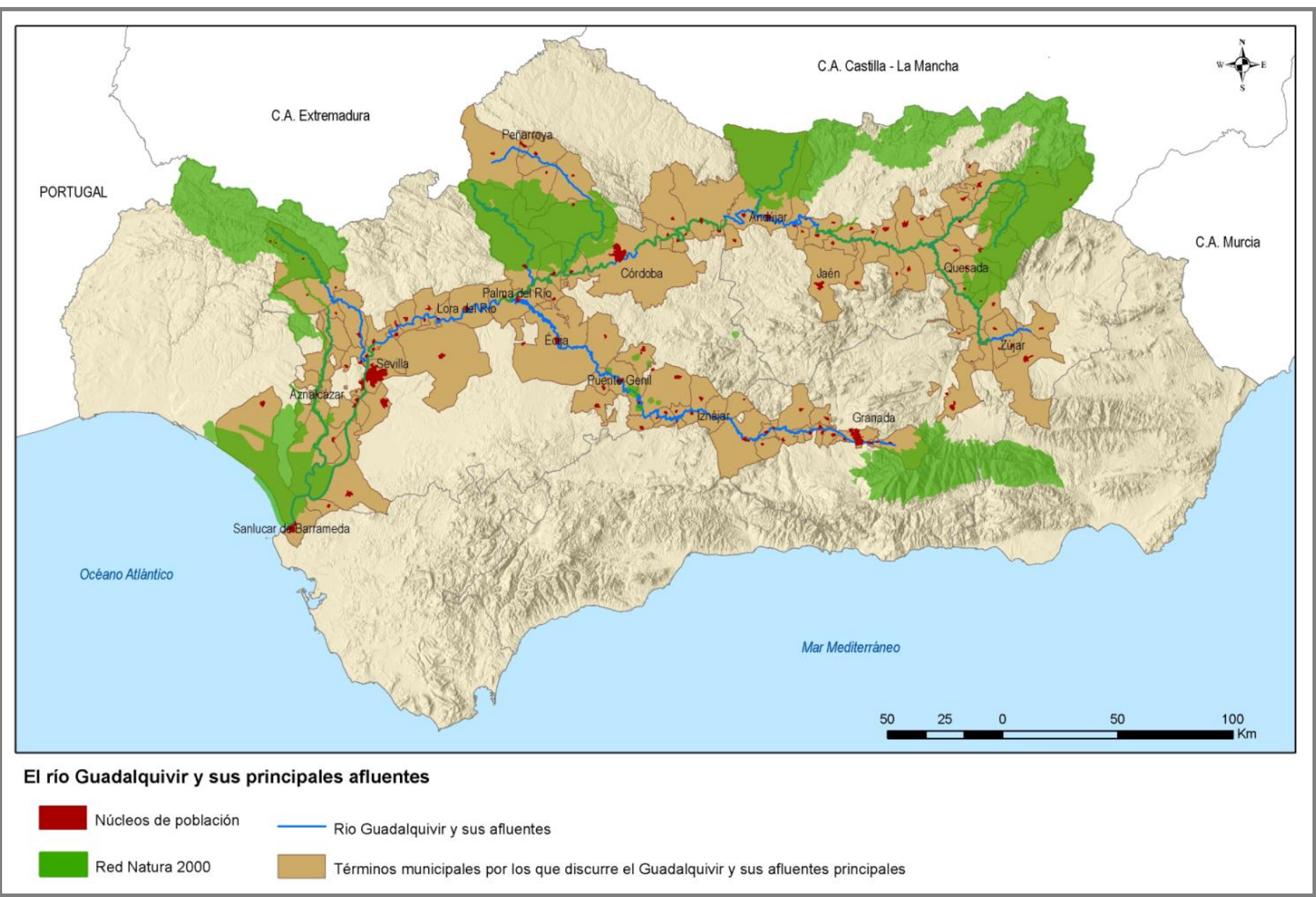

Fuente: Instituto de Desarrollo Regional.

ISSN: 0212-8594 ISSN-e: 2340-2776 № DOI: http://dx.doi.org/10.12795/rea.2011.i28.03

http://www.publius.us.es/estudios andaluces 
Como puede apreciarse, el enlace que este sistema fluvial desempeña con espacios naturales protegidos es muy claro, destacando la presencia de los Parques Nacionales de Doñana y Sierra Nevada, así como Parques Naturales de la Sierra de Aracena y Picos de Aroche, Entorno de Doñana, Sierra de Hornachuelos o Sierra de Cazorla, Segura y las Villas. Desde hace años, la Unión Internacional de Conservación de la Naturaleza viene reivindicando la necesidad de considerar la importancia de los ENP desde un enfoque más funcional que administrativo (visión ecorregional), en su papel de elementos articuladores y dinamizadores del sistema territorial donde se ubican. Así, es preciso comprender la compleja interconexión entre ENP, corredores ecológicos y el resto de la matriz territorial transformada por la acción humana en distintos grados.

\subsection{ENFOQUES DEL TRABAJO.}

La aproximación a un Plan de Acción para el desarrollo sostenible de la red de municipios incluidos en el área socioeconómica del río Guadalquivir y sus afluentes señalados establecía los enfoques necesarios para alcanzar una estrategia equilibrada y respetuosa con las condiciones ambientales de estos espacios.

- Participativo, de implicación socio-institucional.

- Valorizador del patrimonio natural y cultural de estos espacios ribereños, referenciado en la red Natura 2000 y RENPA, y fijando objetivos específicos de acuerdo con sus condiciones y vínculos medioambientales correspondientes.

- Dinamizador socioeconómico del territorio mediante fórmulas de desarrollo sostenible integral compatible con el papel de EPN en el contexto territorial.

- Previsor de las condiciones de protección y de uso y gestión de estos espacios, de acuerdo con las próximas declaraciones de Zonas de Especial Conservación (ZEC), donde proceda.

En resumen, se apreciaba la necesidad de adoptar unos enfoques destinados a la búsqueda de un punto óptimo de equilibrio entre el fuerte aporte de atractivo de los ENP en esta nueva experiencia turística, la figura de un río y eje de dicha estrategia que reunía también la condición de espacio protegido en varios de sus tramos, con las afecciones o limitaciones de uso y aprovechamiento que ello implicaba, y la pretensión de conseguir un desarrollo socioeconómico suficiente para los municipios implicados.

No era posible, por tanto, obviar el papel del componente medioambiental en este proceso estratégico, resultando aconsejable llegar a un consenso en torno a cuestiones esenciales, como la carga máxima de visitantes, las posibilidades y limitaciones de cada parte del territorio beneficiario, o la elaboración de ofertas temáticas acordes con un modelo de actuación en red que no implique necesariamente una homogeneidad o uniformidad del producto turístico creado. 
La siguiente figura refleja fielmente la ubicación de un Lugar de Importancia Comunitaria en el tramo medio del río Guadalquivir, cuyas condiciones de protección y usos limitados requerían adaptar la oferta general del proyecto a unas particularidades bien definidas, cuya vulnerabilidad era considerada "Alta" o "Muy Alta" en un 86\% de su superficie según el riesgo de amenaza de sus hábitats naturales.

De acuerdo con los enfoques planteados, se consideró adecuado adelantar algunas iniciativas o temáticas para debate, cuyo proceso de validación socio-institucional sería determinante para su posterior viabilidad:

- Impulso de un turismo activo y de naturaleza vinculado a las márgenes fluviales: Habilitando ofertas en función de una zonificación adecuada según los tramos del río y la capacidad de carga de los ecosistemas (senderismo, cicloturismo, rutas ecuestres, vuelo en paramotor o en globo aerostático.)

- Desarrollo de actividades relacionadas con la navegación en el río: Ubicación de embarcaderos y disponibilidad de embarcaciones en zonas que reúnan las características para su desarrollo (embarcaciones de recreo en cauce fluvial, cruceros turísticos, deportes acuáticos, etc.).

- Potenciación de un turismo cultural y etnológico: Poniendo en valor elementos del patrimonio cultural material e inmaterial: puentes, molinos, norias, actividades productivas tradicionales asociadas al río y sus expresiones culturales.

- Promoción/concienciación del Espacio-paisaje: Fomentando una interpretación efectiva de los principales valores paisajísticos que conforman la esencia de este Territorio. Visualización del mosaico heterogéneo que conforman los diferentes usos del espacio, alternando con espacios naturales y ecosistemas con distinto grado de madurez.

- Rescate de una gastronomía histórica en declive: Recuperando un recetario histórico casi olvidado y potenciando los productos de la tierra, tradicionales y con denominaciones de origen.

- Lanzamiento de actividades de turismo innovadoras: Compatibilizando un modelo de desarrollo sostenible y alternativo/complementario a las propuestas anteriores.

- Impulso de ECOemprendimientos y ECOformación. Revisando el papel de los nuevos yacimientos de empleo como fórmula adaptable a este modelo de desarrollo asociativo en red. 
Figura 5: Lugar de Importancia Comunitaria Río Guadalquivir - Tramo Medio.

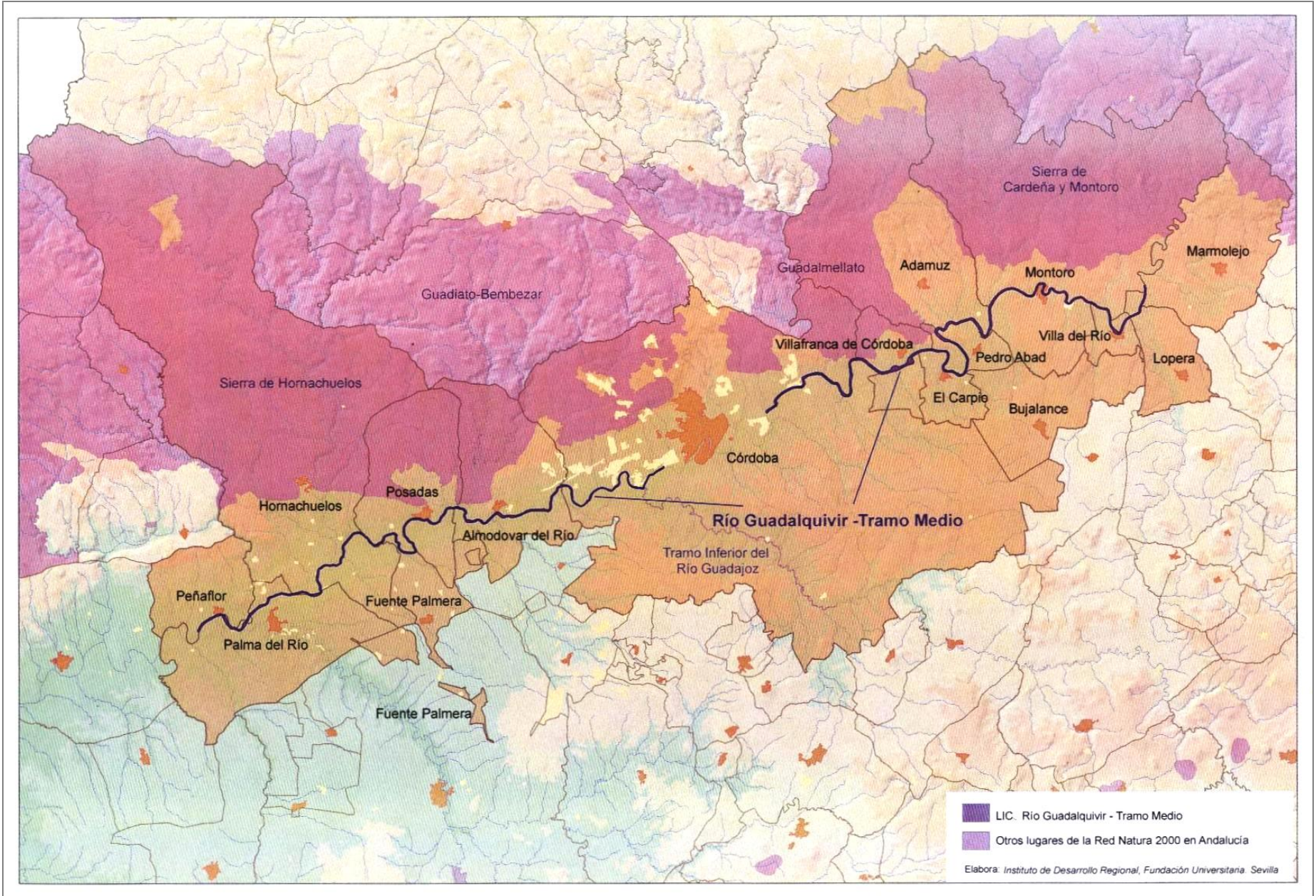

Fuente: Instituto de Desarrollo Regional.

ISSN: 0212-8594 ISSN-e: 2340-2776 № DOI: http://dx.doi.org/10.12795/rea.2011.i28.03

http://www.publius.us.es/estudios andaluces 


\subsection{METODOLOGÍA Y PROGRAMACIÓN DE LA PROPUESTA.}

La propuesta de Plan de Acción se sustentó en una metodología mixta, compuesta de diferentes técnicas o instrumentos de actuación. Algunos de estos soportes metodológicos son los que se exponen a continuación:

- Técnicas cuantitativas de análisis estadístico.

- Entrevistas en profundidad dirigidas a expertos en el ámbito científico y socioinstitucional.

- Talleres de participación y dinamización de agentes claves.

- Sistemas de Información Geográfica (SIG) para territorializar la información sobre un soporte cartográfico de consulta.

- Catalogación de síntesis de recursos, temáticas de interés y potencialidades, y cuestiones sustanciales, como la presencia o grado de incidencia de determinadas políticas, programas o planificación en el territorio beneficiario del Plan de Acción.

Estas técnicas y procesos metodológicos tendrían una programación general para su desarrollo. He aquí un esquema con el que se trabajó de cara a asignar la mayor eficiencia posible al trabajo investigador: 
Figura 6: Esquema de la programación prevista.

\section{Fases}

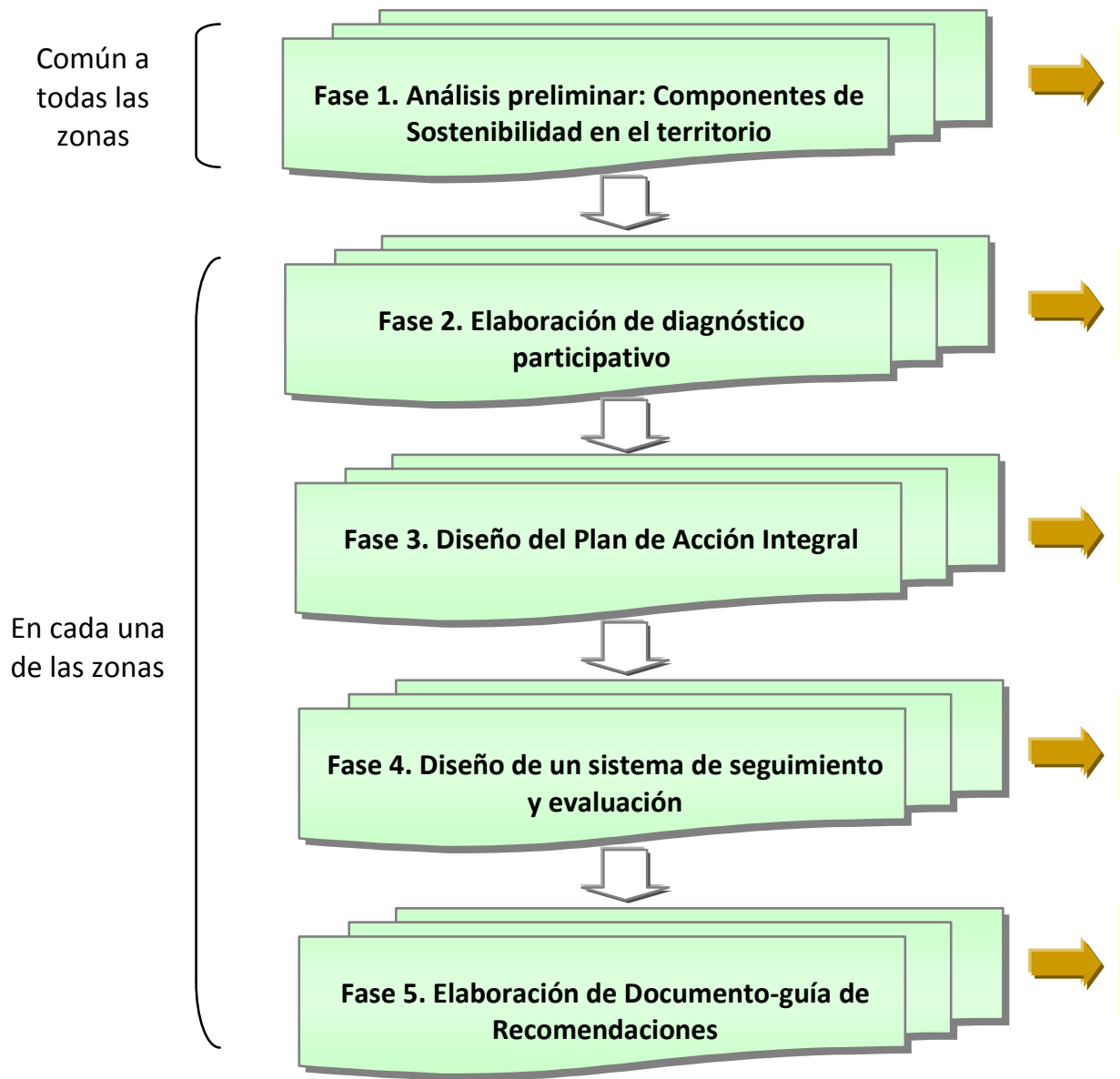

\section{Resultados}

Caracterización del marco socio-institucional económico y medioambiental

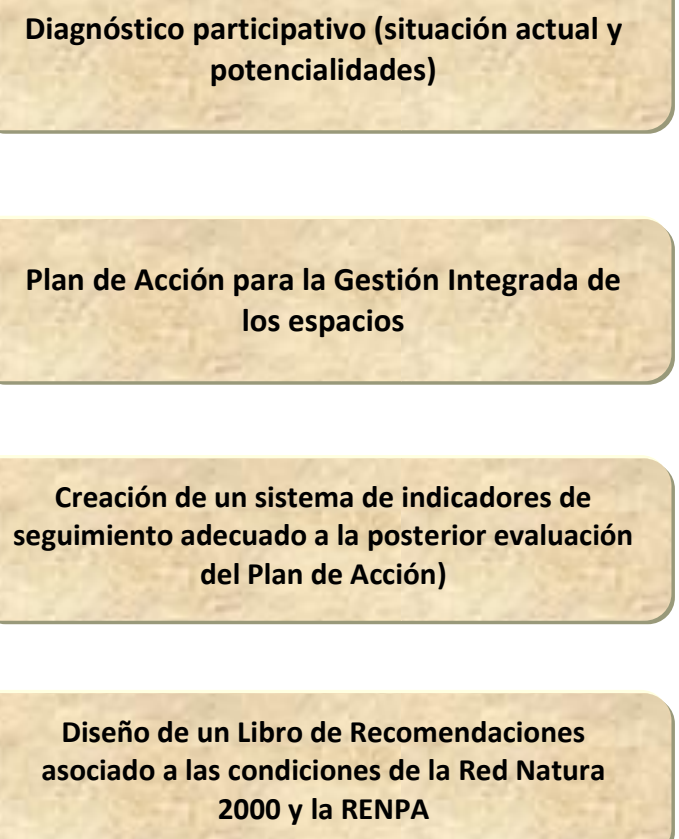

Fuente: Elaboración propia.

ISSN: 0212-8594 ISSN-e: 2340-2776 № DOI: http://dx.doi.org/10.12795/rea.2011.i28.03 


\subsection{RESULTADOS.}

La investigación iniciada en torno a la planificación de una red de desarrollo Local sostenible vinculada al río Guadalquivir y sus principales afluentes quedó finalmente relegada del ámbito de prioridades de las diferentes Administraciones y entidades asociadas en red. Entre las causas más evidentes para su falta de implementación destacaron la pérdida de competencias de la Comunidad Autonómica de Andalucía en la gestión de la Cuenca Hidrográfica del Guadalquivir, al producirse una reversión competencial al Estado Español, además de la intensificación de los efectos de la crisis económica en Andalucía, que obligó a replantear las inversiones del conjunto de entidades y organismos consorciados.

Por todo ello, las labores de investigación acerca de esta posible activación turística sostenible en el ámbito del Guadalquivir y los afluentes seleccionados no superaron la primera fase de análisis, incluyendo así mismo el proceso previo de prospección documental y estadística que dio lugar a la elaboración de esta propuesta.

A continuación, se representa el esquema de trabajo perseguido, cuyos componentes permanecen aún vigentes de cara a una próxima reactivación de esta estrategia asociativa de desarrollo, gestionada bajo criterios de gobernanza, colaboración y coordinación entre sus agentes implicados:

Figura 7: Esquema de Trabajo

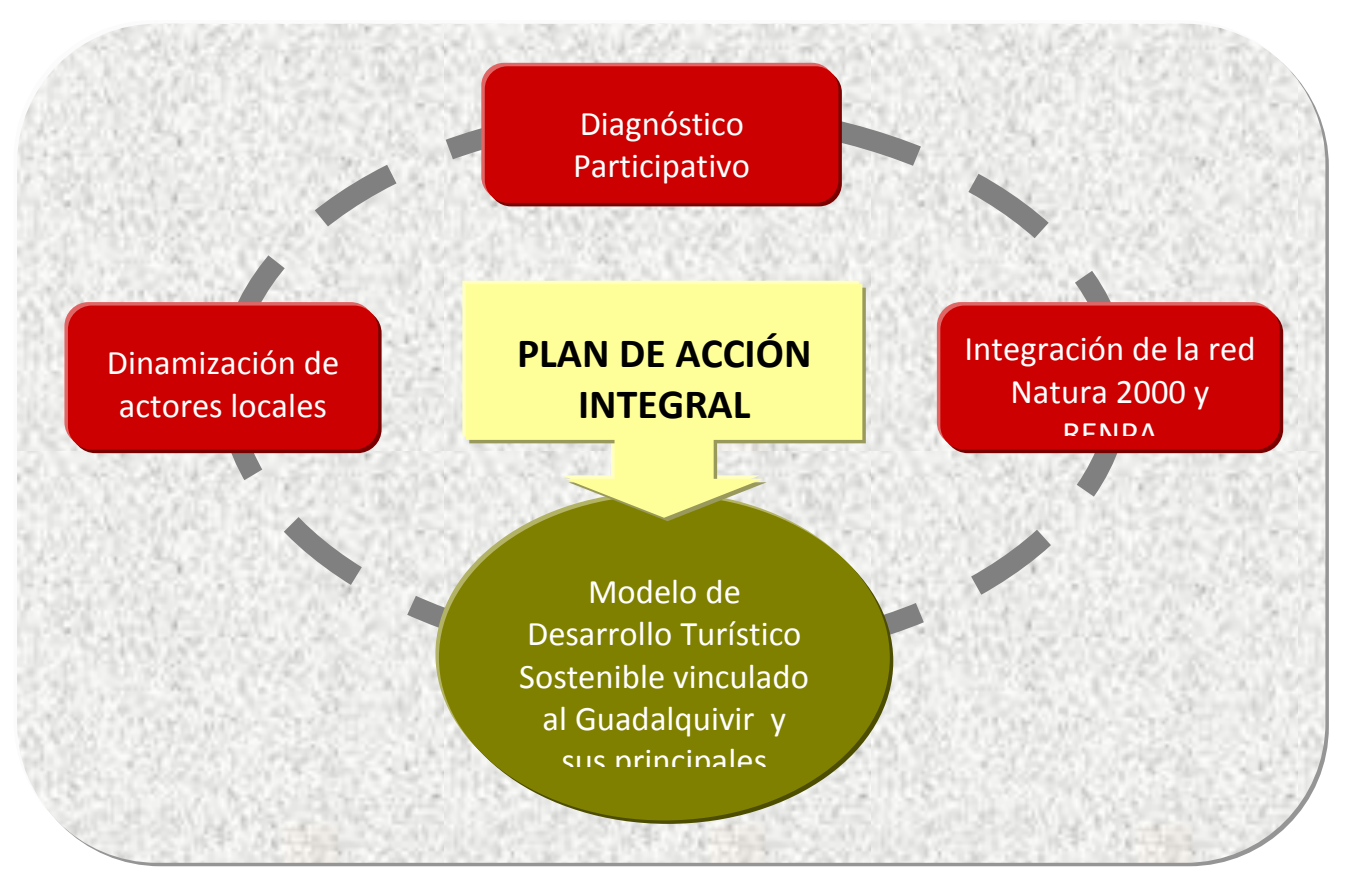

Fuente: Elaboración propia, a partir del Instituto de Desarrollo Regional.

ISSN: 0212-8594 ISSN-e: 2340-2776 № DOI: http://dx.doi.org/10.12795/rea.2011.i28.03

REA 28 (2011):34-54

http://www.publius.us.es/estudios_andaluces 


\section{CONCLUSIONES.}

Existe en Andalucía un espacio sujeto a diferentes iniciativas o figuras de planificación turística que responden a un modelo fraccionado de desarrollo para el principal eje fluvial: el río Guadalquivir.

En los últimos años se ha venido produciendo una concienciación muy importante entre diferentes agentes públicos y privados acerca de las potencialidades turísticas del río Guadalquivir, en su conjunto, comenzando por la creación en 2007 de una Red de Ciudades ribereñas donde el componente municipalista de la iniciativa fue dominante. Un año más tarde la idea de realizar una dinamización socioeconómica de este ámbito basada en la promoción turística fue marcada como una prioridad para la Administración Autonómica de Andalucía, planteando el Proyecto Guadalquivir Turístico como la figura emblemática de ese reto.

Después de un proceso de reflexión y de búsqueda de adhesiones a esta idea, se estima conveniente la creación de un Consorcio de promoción turística integrado por la Junta de Andalucía, las diputaciones provinciales de Cádiz, Córdoba, Granada, Huelva y Sevilla, la Confederación de Empresarios de Andalucía, los sindicatos CCOO-A y UGT-A y la Red de Ciudades Río Guadalquivir (mayo de 2011). Además se añade el eje fluvial del río Genil, como principal afluente del Guadalquivir, conformando la denominada "Y andaluza".

La dificultad de encajar las diferentes iniciativas turísticas territoriales implementadas hasta la fecha mediante el exclusivo nexo del río, así como la puesta en marcha de una nueva estrategia global e integradora de la diversidad de municipios y territorios adyacentes a estos dos ejes fluviales, fue interpretada por parte del Instituto de Desarrollo Regional como una oportunidad si se vinculaba a alguna variable suficientemente sólida, con atractivo demostrado en la promoción turística de interior y con presencia suficiente en el conjunto de los territorios beneficiarios. De este modo, se escogió la variable medioambiental, al existir una presencia muy destacada de espacios naturales protegidos en los propios ríos Guadalquivir y Genil, así como en su entorno más inmediato. Se estimó conveniente considerar la figura del Corredor Ecológico como soporte funcional de estos ríos, añadiendo a modo de prueba otros afluentes igualmente importantes, como la Ribera de Huelva, el Jándula o el Guadiana Menor.

Tras disponer de un soporte territorial más amplio y en conexión con espacios naturales protegidos de enorme relevancia en Andalucía, como los Parques Naturales de Hornachuelos y Cazorla, Segura y las Villas, o los Parques Nacionales de Doñana y Sierra Nevada, se consideró adecuado implementar una investigación vinculada al proceso de diseño de un Plan de Acción Integral para el desarrollo turístico del valle del Guadalquivir y sus afluentes principales, aplicando una metodología mixta, con un

ISSN: 0212-8594 ISSN-e: 2340-2776 № DOI: http://dx.doi.org/10.12795/rea.2011.i28.03

REA 28 (2011):34-54

http://www.publius.us.es/estudios_andaluces 
especial protagonismo del diagnóstico participativo y la dinamización de actores locales.

No obstante, los trabajos iniciales resultaron ralentizados y finalmente detenidos ante la pérdida de competencias gestoras de la Cuenca Hidrográfica del Guadalquivir por parte de la Administración Autonómica de Andalucía y los devastadores efectos de la crisis, que han contraído sensiblemente la capacidad inversora del conjunto de entidades consorciadas. Aunque se ha producido un severo colapso del proceso investigador, es evidente la posibilidad de una próxima reanudación, en concordancia con el interés que suscita el impulso socioeconómico del ámbito geográfico considerado.

Queda aquí recogido el testimonio de quien ha participado en la dinámica constitutiva de la Red de Ciudades del Río Guadalquivir, sus primeros pasos y expectativas, y su posterior desarrollo en forma de proyecto común interinstitucional. Los esfuerzos desarrollados hasta la fecha no han sido baldíos, sino que han supuesto todo un ejemplo de voluntad integradora de entidades y sujetos diferentes unidos por la esperanza de un desarrollo socioterritorial equilibrado y participativo en torno a la mayor arteria fluvial de Andalucía.

\section{BIBLIOGRAFÍA}

Alburquerque, F. (2002): Guía para agentes. Desarrollo económico territorial. Instituto de Desarrollo Regional. Fundación Universitaria. Sevilla, 214 páginas.

Alburquerque, F.; Delgadillo, J. (2009): Emprendimientos de base ecológica en las áreas de influencia socioeconómica de los Parques Naturales de Andalucía. Cuadernos del IDR nㅇ 40. Instituto de Desarrollo Regional. Fundación Universitaria. Sevilla, 98 páginas.

Cantarero, J.M. (2001): Planificación y gestión del turismo en el medio rural. Centro de Turismo Interior de Andalucía. Consejería de Turismo y Deporte. Junta de Andalucía. Úbeda (Jaén), 272 páginas.

Contreras G.A. y Jiménez, E. (2010): Plan de Acción para el Desarrollo Rural Sostenible vinculado al río Guadalquivir y sus principales afluentes. Instituto de Desarrollo Regional. Fundación Universitaria. Documento inédito. Sevilla, 15 páginas.

Fernández Latorre, F. M. (2012): Formación, Investigación e Innovación en Turismo. Digital@tres editores. Sevilla.

Fundación Centro Español de Estudios de América Latina (1997): El enfoque del Marco Lógico. Manual para la planificación de proyectos orientada mediante objetivos. Instituto Universitario de Desarrollo y Cooperación. Universidad Complutense de

ISSN: 0212-8594 ISSN-e: 2340-2776 № DOI: http://dx.doi.org/10.12795/rea.2011.i28.03

REA 28 (2011):34-54

http://www.publius.us.es/estudios_andaluces 
Gustavo A. Contreras Cabrera

Asociacionismo en red para la planificación del desarrollo turístico sostenible. El caso del río Guadalquivir y sus principales afluentes

Madrid y Fundación Centro Español de Estudios de América Latina - CEDEAL. Madrid, 115 páginas.

Galiana, L. y Vinuesa, J. (2010): Teoría y práctica para una ordenación racional del territorio. Espacios y Sociedades, Serie Mayor. Editorial Síntesis. Madrid, 346 páginas.

Galindo, L. (2008): Desarrollo territorial en la Vega Media de Sevilla: Elementos de un tejido empresarial urbano-rural. Revista de Estudios Andaluces, no 27, pp. 155-203

García, M.R. y Montes, c. (2003): Vínculos en el paisaje mediterráneo. El papel de los espacios protegidos en el contexto territorial. Consejería de Medio Ambiente (Junta de Andalucía). Sevilla, 216 páginas.

Palenzuela, P. y otros (2000): Guía para la puesta en valor del patrimonio en el medio rural andaluz. Consejería de Agricultura y Pesca - Empresa Pública para el Desarrollo Agrario y Pesquero de Andalucía (DAP). Junta de Andalucía. Córdoba, Varios volúmenes.

Pozuelo, I y otros (2004): Guía turística de la provincia de Sevilla. Descubrir un destino. Turismo de la Provincia. Diputación de Sevilla. Sevilla, 152 páginas.

ISSN: 0212-8594 ISSN-e: 2340-2776 № DOI: http://dx.doi.org/10.12795/rea.2011.i28.03 